\title{
Expression of Complement Receptor Type 1 on Erythrocytes in Autoimmune Diseases
}

\author{
Deng-Ho Yang ${ }^{1,2}$, Chen-Hung Chen ${ }^{3}$, Cheng-Chung Wei ${ }^{1,4}$ and Ya-Wen Cheng ${ }^{1,5 *}$ \\ 1 Institute of Medicine, Chung Shan Medical University, Taiwan \\ ${ }^{2}$ Division of Rheumatology/Immunology/Allergy, Department of Internal Medicine, Taichung Armed-Forces General Hospital, Taiwan \\ ${ }^{3}$ Division of Rheumatology/Immunology/Allergy, Department of Internal medicine, Tri-Service General Hospital, National Defense Medical Center, Taiwan \\ ${ }^{4}$ Division of Rheumatology/Immunology/Allergy, Department of Internal Medicine, Chung Shan Medical University Hospital, Taichung Taiwan \\ ${ }^{5}$ Graduate Institute of Cancer Biology and Drug Discovery, Taipei Medical University, Taipei, Taiwan
}

\begin{abstract}
Objective: The expression of complement receptor type 1 on different cells is associated with autoimmunity. Erythrocyte-Complement Receptor Type 1 (E-CR1) is a candidate for early diagnosis of Systemic Lupus Erythematosus (SLE) and assessed the roles of disease activity. We evaluated the expression of E-CR1 in the patients with SLE and other autoimmune diseases.

Methods: We conducted a cross-sectional investigation of 3 groups: (1) 36 patients with SLE; (2) 51 patients with other diseases including Rheumatoid Arthritis (RA), Sjögren's syndrome, anti-phospholipid syndrome, Mixed Connective Tissue Disease (MCTD), Raynaud's disease, ankylosing spondylitis, pulmonary Tuberculosis (TB); and (3) 26 healthy controls. Erythrocytes were analyzed by flow cytometry to determine levels of E-CR1.

Results: We found a significant reduction in the mean levels of E-CR1 in SLE patients compared to patients with healthy controls $(1.79 \pm 0.16$ versus $3.82 \pm 0.32, P<0.05)$. However, in patients with RA, MCTD, and TB, decreased E-CR1 levels were also observed. There was a statistically significant correlation between reduced levels of E-CR1 and SLE disease activity index $(r=-0.326, P<0.05)$. The E-CR1 levels were inversely correlated with urine daily protein loss $(r=-0.364, P<0.05)$

Conclusions: Although E-CR1 levels may be a useful diagnostic marker for SLE, a possible limitation is that no significant differences in E-CR1 levels were observed among patients with SLE, those with MCTD and TB. Overall, E-CR1 levels in SLE patients are related to disease activity index and reflect renal clearance through urine daily protein loss.
\end{abstract}

Keywords: Systemic lupus erythematosus; Rheumatoid arthritis; Mixed connective disuse disease; Tuberculosis; Complement receptor type 1; Erythrocyte; Biomarker

\section{Introduction}

Complement Receptor Type 1 (CR1) is a transmembrane multifunctional glycoprotein that plays a crucial role in the clearance of Immune Complexes (ICs) from the blood circulation; it is expressed on various cell types, including neutrophils, granulocytes, erythrocytes, B lymphocytes, T lymphocytes, follicular dendritic cells, macrophages, and monocytes [1,2]. More than $85 \%$ of CR1 in blood is present on erythrocytes [1]. Systemic Lupus Erythematosus (SLE) is a chronic systemic autoimmune disease with circulating autoantibodies, typically against self-antigens related to the nuclei [3]. The autoimmune mechanisms of autoantibodies and forming pathologic ICs may target diverse tissues and organs to induce damage. Numerous genetic and environmental factors can induce the development of SLE. Thus, abnormalities in complement activation and clearance of circulating ICs are important in the pathogenesis of SLE [4]. Multi-organ inflammatory injury in SLE appears to be caused by tissue-deposition of ICs consisting of autoantigens, autoantibodies and activated complement. This process can elicit a subsequent "cytokine storm" $[5,6]$.

The reduced expression of CR1 is found in the patients with SLE and associated with autoimmune dysregulation. This defect presentation of CR1 can be seen in erythrocytes, B lymphocytes, leukocytes. Abnormal formation of ICs and increased circulating autoantibodies may develop under the low expression of CR1 in SLE. A previous study revealed that a reduction in Erythrocyte-Complement Receptor Type 1 (E-CR1) is associated with lupus nephritis $[7,8]$. In this study, we evaluated the circulating levels of E-CR1 in SLE patients and other autoimmune diseases patients.

\section{Materials and Methods}

\section{Study participants}

All study participants were 18 years of age or above, and each provided a written informed consent. None of the patients were excluded from participation on the basis of sex or ethnicity. This study was approved by the Tri-Service General Hospital Institutional Review Board (Grant no: 098-05-084) based on the declaration of Helsinki.

\section{SLE patients}

Blood samples were collected from 36 SLE patients who met the 1982 ACR revised criteria for the classification of definite SLE. This

*Corresponding author: Ya-Wen Cheng, Institute of Medicine, Chung Shan Medical University, No.110, Sec.1, Jianguo N.Rd., Taichung City 40201, Taiwan, Tel: +886-4-24730022; Fax: +886-4-24723229; E-mail: deng6263@ms71.hinet.net

Received December 13, 2013; Accepted Januauary 15, 2014; Published Januauary 22, 2014

Citation: Yang DH, Chen CH, Wei CC, Cheng YW (2014) Expression of Complement Receptor Type 1 on Erythrocytes in Autoimmune Diseases. J Mol Biomark Diagn 5: 163. doi:10.4172/2155-9929.1000163

Copyright: ( 2014 Yang DH, et al. This is an open-access article distributed under the terms of the Creative Commons Attribution License, which permits unrestricted use, distribution, and reproduction in any medium, provided the original author and source are credited 
Citation: Yang DH, Chen CH, Wei CC, Cheng YW (2014) Expression of Complement Receptor Type 1 on Erythrocytes in Autoimmune Diseases. J Mol Biomark Diagn 5: 163. doi:10.4172/2155-9929.1000163

Page 2 of 6

group of patients consisted of 33 women and 3 men with age ranging from 18 to 77 years (mean age $=38$ years). Disease activity was evaluated in each patient according to the Systemic Lupus Erythematosus Disease Activity Index (SLEDAI). E-CR1 levels were measured by flow cytometry. Serum levels of anti-dsDNA, C3, C4, white blood count, hemogloblin, platelets, Blood Urea Nitrogen (BUN), creatinine (Cr), C-Reactive Protein (CRP), and urine Daily Protein Loss (DPL) were also assessed.

\section{Patients with other diseases}

Fifty one randomly selected patients with various other rheumatic, autoimmune, or infectious diseases were recruited. The other diseases included Rheumatoid Arthritis (RA, used 2010 ACR / EULAR rheumatoid arthritis classification criteria), Sjögren's Syndrome (SS, used American-European consensus Sjögren's classification criteria), Anti-Phospholipid Syndrome (APS, used Sydney criteria), Mixed Connective Tissue Disease (MCTD, used Alarcon-Segovia diagnostic criteria), Raynaud's Disease (RD, used Allen Brown criteria), Ankylosing Spondylitis (AS, used modified New York criteria), and Pulmonary Tuberculosis (TB, positive sputum culture).

\section{Healthy controls}

Twenty-six healthy individuals were recruited as controls. These participants were required to complete a brief questionnaire regarding previous or current medical conditions.

\section{Flow cytometric characterization of erythrocytes}

For each assay, $1 \mathrm{~mL}$ of blood was collected from every participant. Blood samples were placed in a Vacutainer tube containing EDTA. Five microlitres of whole blood was removed and incubated with $50 \mu \mathrm{L}$ of anti-CR1 monoclonal antibody $2 \mathrm{~B} 11(5 \mu \mathrm{g} / \mathrm{mL})$ at a $1: 250$ dilution. After incubation, cells were washed twice with $1 \mathrm{~mL}$ of diluent buffer and centrifuged at $1500 \mathrm{~g}$ for 3 minutes at $4^{\circ} \mathrm{C}$. One microlitre of Fluorescein Isothiocyanate (FITC)-conjugated goat anti-mouse immunoglobulin-specific polyclonal antibody (BD Pharmingen ${ }^{\mathrm{mm}}$, NJ, USA; $500 \mu \mathrm{g} / \mathrm{mL}$ ) was added to the supernatant for 30 minutes at $4^{\circ} \mathrm{C}$. Cells were then washed again as previously described and resuspended in $1 \mathrm{~mL}$ Phosphate Buffered Saline (PBS). The samples were analyzed by flow cytometry using a FACSCalibur flow cytometer (BD Immunocytometry System, San Jose, CA). Erythrocytes were electronically gated for 30,000 cells on the basis of their forward and side scatter properties. Surface expression of CR1 on gated cells was detected by specific Mean Fluorescence Intensity (sMFI) (CR1-specific mean fluorescence minus the isotype control mean fluorescence).

\section{Statistical analysis}

SPSS version 15.0 software (SPSS Inc., Chicago, IL, USA) was used to perform all statistical analyses. Differences between the median values of defined patient groups were compared using the nonparametric Mann-Whitney U test. A Spearman's rank correlation was used to detect correlations among different study parameters. A $P$ value of less than 0.05 was considered statistically significant.

\section{Results}

\section{Characteristics of the three study groups}

The study population consisted of 36 patients with SLE, 51 patients with other diseases, and 26 healthy controls. The SLE patients had a mean age of $38.3 \pm 2.6$ years ( 33 women and 3 men) and were divided into 4 subgroups as follows: SLE without nephritis or hemolytic anemia, SLE with nephritis, SLE with Chronic Renal Failure (CRF), and SLE with hemolytic anemia. The laboratory data and SLEDAI are shown in Table 1. Fifty-one patients identified to have various types of other disease were distributed as follows: RA ( $n=11)$, SS $(n=12)$, APS $(\mathrm{n}=3), \operatorname{MCTD}(\mathrm{n}=5), \mathrm{RD}(\mathrm{n}=4), \operatorname{AS}(\mathrm{n}=11)$, and TB $(\mathrm{n}=5)$. The healthy controls had a mean age of $31.5 \pm 2.2$ years (18 women and 8 men). The patients with other disease and healthy controls had normal renal function.

\section{E-CR1 levels in controls, SLE patients, and patients with other diseases}

The SLE patients showed significantly lower levels of E-CR1 relative to healthy controls and patients diagnosed with RA, SS, APS, $\mathrm{RD}$, and AS (Figure 1). In patients diagnosed to have RA, MCTD, and TB, significantly lower levels of E-CR1 were found than that in healthy controls $(2.71 \pm 0.46,2.28 \pm 0.52,1.83 \pm 0.43$ versus $3.82 \pm 0.32$, $P<0.05)$. Levels of E-CR1 were significantly lower in SLE patients than in patients with RA ( $1.79 \pm 0.16$ versus $2.71 \pm 0.46, P=0.036)$. Reduced levels of E-CR1 were found in SLE, MCTD, and TB patients, but the differences among these groups were not statistically significant $(1.79$ \pm 0.16 versus $2.28 \pm 0.52, P=0.377$, and $1.79 \pm 0.16$ versus $1.83 \pm 0.43$, $P=0.727$ ). The mean sMFI values for SLE patients, patients with other diseases, and healthy controls are shown in Table 2. In SLE patients, there was a statistically significant correlation between reduced levels of E-CR1 and SLEDAI ( $\mathrm{r}=-0.326, P<0.05$, Figure 2). No correlation was detected between E-CR1 and levels of anti-dsDNA, C3 or C4. The E-CR1 levels were inversely correlated with urine DPL $(r=-0.364$, $P<0.05$, Figure 3 ).

\begin{tabular}{|c|c|c|c|c|}
\hline Laboratory manifestations & $\begin{array}{l}\text { SLE without renal disease or hemolytic } \\
\text { anemia }(n=19)\end{array}$ & SLE with nephritis $(n=11)$ & SLE with CRF $(n=2)$ & SLE with hemolytic anemia $(n=4)$ \\
\hline Anti-dsDNA (IU/mL) & $87 \pm 15$ & $124 \pm 11$ & $47 \pm 6$ & $78 \pm 54$ \\
\hline $\mathrm{C} 3(\mathrm{mg} / \mathrm{dL})$ & $74 \pm 5$ & $53 \pm 10$ & $74 \pm 6$ & $53 \pm 7$ \\
\hline $\mathrm{C} 4(\mathrm{mg} / \mathrm{dL})$ & $14 \pm 6$ & $9 \pm 8$ & $20 \pm 10$ & $6 \pm 2$ \\
\hline White blood cell /uL & $4.332 \pm 410$ & $6,303 \pm 510$ & $9,650 \pm 2,771$ & $6,302 \pm 570$ \\
\hline Hemoglobulin (g/dL) & $10.7 \pm 0.9$ & $11 \pm 0.8$ & $9 \pm 0.6$ & $8.2 \pm 0.75$ \\
\hline Platelet /uL & $195,444 \pm 12,299$ & $211,636 \pm 14,271$ & $245,000 \pm 12,231$ & $167,750 \pm 27,211$ \\
\hline BUN (mg/dL) & $14.4 \pm 1.9$ & $24.1 \pm 2.9$ & $94.5 \pm 5.85$ & $14.7 \pm 1.28$ \\
\hline $\mathrm{Cr}(\mathrm{mg} / \mathrm{dL})$ & $0.6 \pm 0.06$ & $1.0 \pm 0.16$ & $9.2 \pm 1.85$ & $0.6 \pm 0.07$ \\
\hline $\mathrm{DPL}(\mathrm{mg})$ & $288 \pm 399$ & $5,167 \pm 1,551$ & $3,010 \pm 1,391$ & $1,773 \pm 1,032$ \\
\hline CRP (mg/dL) & $0.5 \pm 0.08$ & $1.2 \pm 0.07$ & $0.4 \pm 0.04$ & $0.1 \pm 0.05$ \\
\hline SLEDI & $6.74 \pm 1.21$ & $13.18 \pm 2.71$ & $15 \pm 3.71$ & $5.25 \pm 2.62$ \\
\hline
\end{tabular}

$\mathrm{SLE}=$ Systemic Lupus Erythematosus; $\mathrm{CRF}=$ Chronic Renal Failure; $\mathrm{DPL}=$ Daily Protein Loss

Table 1: Laboratory manifestations of SLE patients. 
Citation: Yang DH, Chen CH, Wei CC, Cheng YW (2014) Expression of Complement Receptor Type 1 on Erythrocytes in Autoimmune Diseases. J Mol Biomark Diagn 5: 163. doi:10.4172/2155-9929.1000163

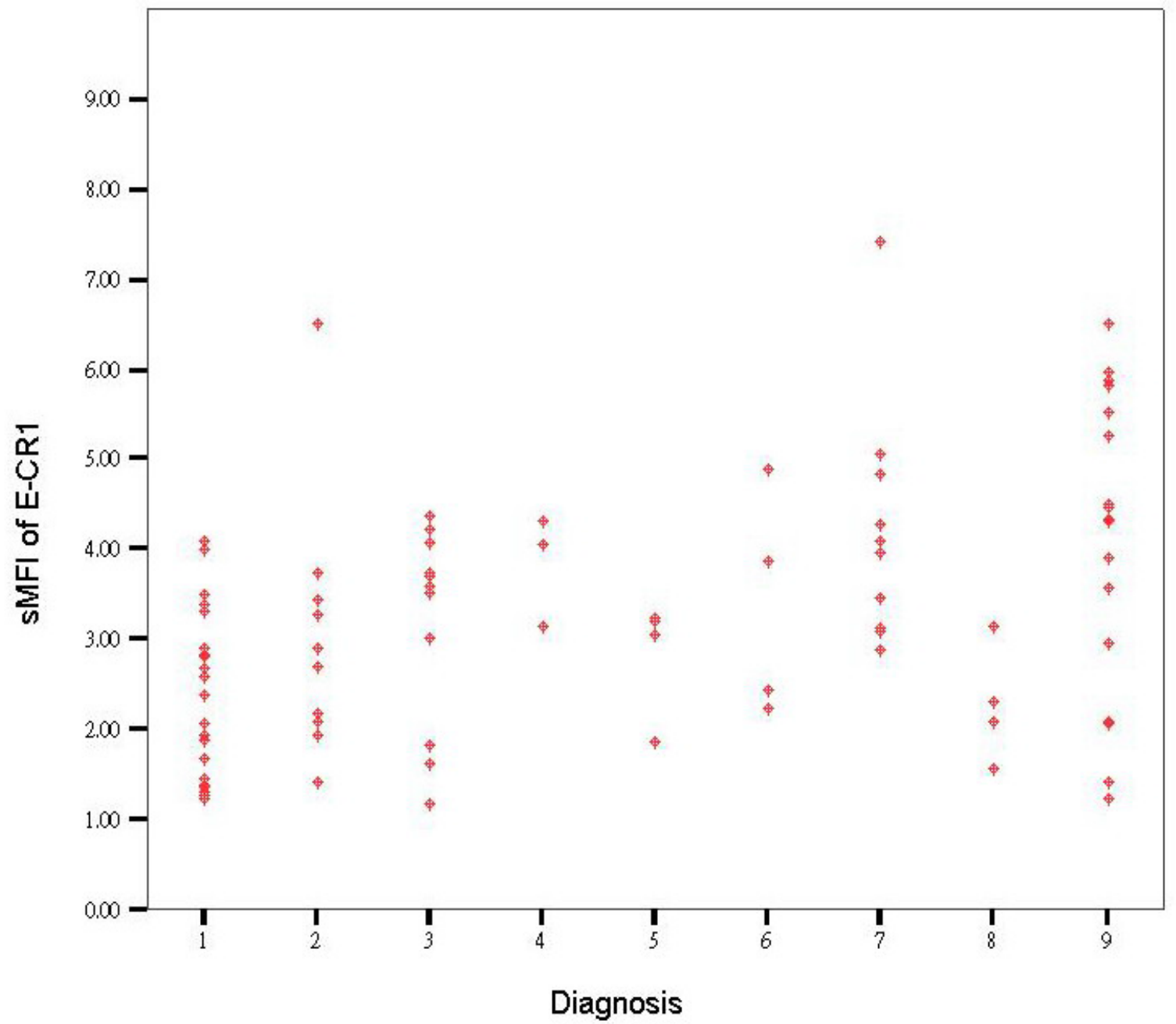

1= Systemic Lupus Erythematosus, 2= Rheumatoid Arthritis, 3= Sjögren's Syndrome, 4= Anti-Phospholipid Syndrome, 5= Mixed Connective Tissue Disease, 6= Raynoud's disease, $7=$ Ankylosing Spondylitis, $8=$ Pulmonary Tuberculosis, $9=$ Healthy Controls

Figure 1: Levels of E-CR1 in different diseases.

\begin{tabular}{|c|c|}
\hline Subjects & Mean sMFI of E-CR1 \\
\hline SLE $(n=36)$ & $1.79 \pm 0.16^{\text {ab }}$ \\
\hline RA $(n=11)$ & $2.71 \pm 0.46^{\text {a }}$ \\
\hline SS $(n=12)$ & $3.06 \pm 0.31$ \\
\hline APS $(n=3)$ & $3.75 \pm 0.35$ \\
\hline MCTD $(n=5)$ & $2.28 \pm 0.52^{\text {a }}$ \\
\hline RD $(n=4)$ & $3.26 \pm 0.63$ \\
\hline AS $(n=11)$ & $3.83 \pm 0.49$ \\
\hline TB $(n=5)$ & $1.83 \pm 0.43^{\text {ab }}$ \\
\hline Healthy controls $(n=26)$ & $3.82 \pm 0.32$ \\
\hline
\end{tabular}

aSignificant difference $(P<0.05)$ compared to healthy controls.

bSignificant difference $(P<0.05)$ compared to RA patients.

$\mathrm{SLE}=$ Systemic Lupus Erythematosus, RA= Rheumatoid Arthritis, SS= Sjögren's Syndrome, APS= Anti-Phospholipid Syndrome, MCTD= Mixed Connective Tissue Disease, RD= Raynoud's Disease, AS= Ankylosing Spondylitis, TB= Pulmonary Tuberculosis

Table 2: Mean SMFI values of E-CR1 in SLE patients, patients with other diseases, and healthy controls.

\section{E-CR1 levels in different subgroups of SLE patients}

Significantly lower mean levels of E-CR1 were found in SLE patients with nephritis than in SLE patients with CRF or hemolytic anemia $(1.25 \pm 0.18$ versus $3.69 \pm 0.31,2.45 \pm 0.56, P<0.05)$. The mean levels of E-CR1 in SLE patients with nephritis were lower than that in SLE patients without nephritis, but the difference was not statistically significant $(1.25 \pm 0.18$ versus $1.76 \pm 0.20, P=0.102)$. In the subgroup of SLE patients with CRF, the levels of E-CR1 were not significantly 


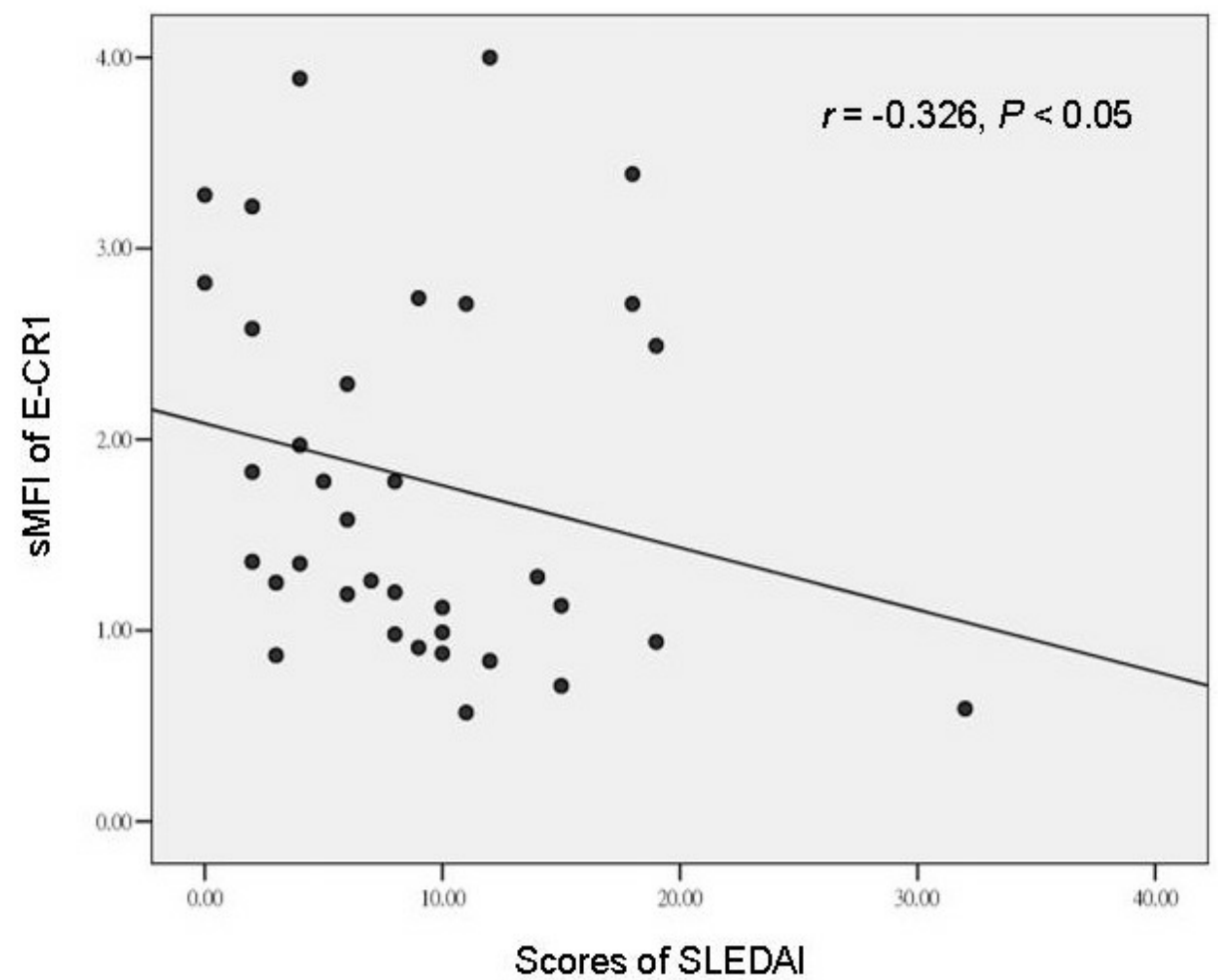

Figure 2: Correlation between SMFI values for E-CR1 and SLEDAI at the time of analysis.

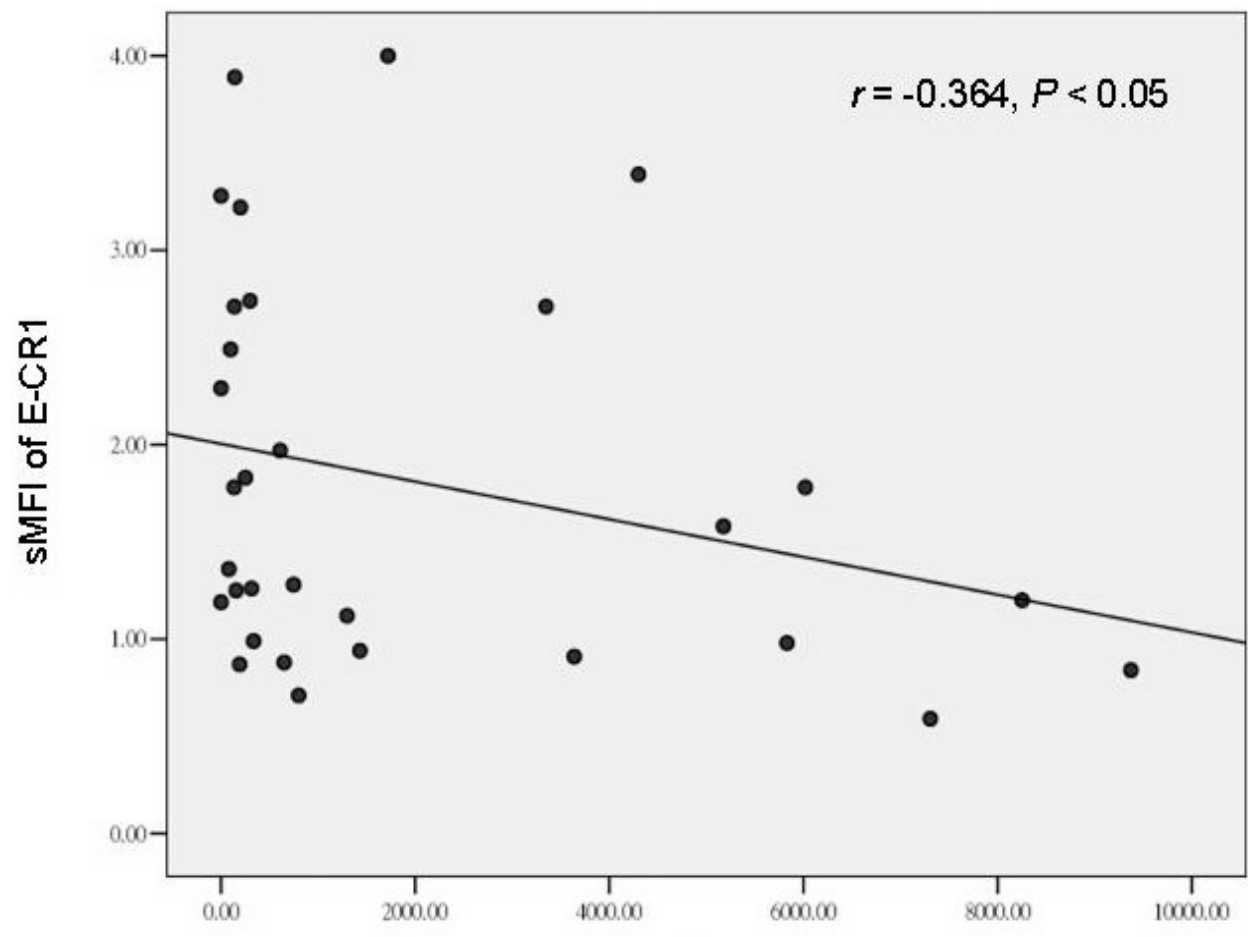

Urine daily protein loss $(\mathrm{mg})$

Figure 3: Correlation between sMFI values for E-CR1 and urine daily protein loss at the time of analysis. 
lower than those of healthy controls $(3.69 \pm 0.31$ versus $3.82 \pm 0.32$, $P=0.5)$. In the subgroup of SLE patients with hemolytic anemia, the levels of E-CR1 were not significantly decreased compared to those of healthy controls $(2.45 \pm 0.56$ versus $3.82 \pm 0.32, P=0.089)$. The mean MFI values for E-CR1 in the 4 subgroups of SLE patients are shown as Table 3 .

\section{Discussion}

CR1 contains 30 Short Consensus Repeats (SCRs) that are organized into 4 groups from SCR1 to SCR28 [1]. Different protein can bind the different site of CR1 to present different biological function [2-4]. CR1 is a multifunctional polymorphic glycoprotein, including cell surface and nonmembrane bound soluble forms. It expressed on erythrocytes has an important role in complement pathway regulation and clearance of ICs, while CR1 expressed on polymorphonuclear cells and monocytes is involved in inducing phagocytosis [3]. When expressed on B cells, CR1 appears to be involved in controlling proliferation of these cells $[5,6]$. Several anti-E-CR1 monoclonal antibodies with binding specificity for SCR1 to SCR28 have been used in preliminary studies for the evaluation of autoimmune disorders and infectious diseases associated with E-CR1. Here, we used the specific anti-E-CR1 monoclonal antibody, 2B11, which binds within the SCR29-30 region of CR1. CR1-2B11 enables more accurate quantification of E-CR1; further, compared to other anti-E-CR1 monoclonal antibodies, it forms more stable complexes that are likely to be resistant to degradation during storage or handling of erythrocytes [7].

In patients with SLE, lower levels of CR1 have been found on various cells, including erythrocytes, leukocytes, neutrophils, reticulocytes, and B cells [8-10]. Circulating E-CR1 can bind with complement and affect the disease activity of SLE [11]. In addition to SLE, E-CR1 levels are decreased in other pathological conditions such as hemolytic anemia, HIV infection, malaria, lepromatous leprosy, tuberculosis, and RA [4,12-15]. The formation of ICs is an important mechanism of the adaptive immune response that promotes removal of foreign or self antigens. In this process, complements maintain solubility of the ICs via complement receptors, and clearance by phagocytosis occurs through mechanisms involving $\mathrm{F} c \gamma$ receptors $[16,17]$. With increasing levels of circulating ICs, reductions in E-CR1 levels occur in patients with pulmonary TB [15]. In SLE, E-CR1 is the major vehicle for the clearance of circulating ICs [18]. E-CR1-mediated clearance of ICs is involved in the pathogenic mechanism of these autoimmune or chronic infectious disorders. A previous study revealed that abnormally high levels of E-C4d and low levels of E-CR1 are useful for the diagnosis of SLE because of their high sensitivity and specificity for lupus [19]. In SLE patients with active nephritis, lower numbers of E-CR1 are found than in SLE patients who do not have clinically apparent renal disease [8]. In this present study, significant reductions in sMFI values for E-CR1 were observed in SLE patients compared to those in healthy controls; this finding was consistent with those reported in previous studies $[8,10,19]$. In SLE patients with renal problems, a reduction in

\begin{tabular}{|c|c|}
\hline SLE patients & Mean sMFI of E-CR1 \\
\hline SLE $(n=19)$ & $1.76 \pm 0.20^{\mathrm{a}}$ \\
\hline SLE with nephritis $(\mathrm{n}=11)$ & $1.25 \pm 0.18^{\mathrm{a}}$ \\
\hline SLE with CRF $(\mathrm{n}=2)$ & $3.69 \pm 0.31^{\mathrm{b}}$ \\
\hline SLE with hemolytic anemia $(\mathrm{n}=4)$ & $2.45 \pm 0.56^{\mathrm{c}}$ \\
\hline
\end{tabular}

aSignificant difference $(P<0.05)$ compared to healthy controls.

bNo Significant difference $(P=0.5)$ compared to RA patients.

cNo significant difference $(P=0.089)$ compared to healthy controls.

Table 3: Mean SMFI values of E-CR1 in SLE patients.
sMFI values for E-CR1 was found compared to SLE patients without significant nephritis. This finding could be linked with a significant inverse correlation between urine DPL and sMFI values for E-CR1 (Figure 3, $\mathrm{r}=-0.364, \mathrm{P}<0.05$ ). The levels of $\mathrm{E}-\mathrm{CR} 1$ in SLE patients also correlated inversely with SLEDAI (Figure $2, \mathrm{r}=-0.326, \mathrm{P}<0.05$ ). Because the number of SLE patients was small, the relationship was not strong, but there was still a statistically significant. This result may be due to significantly greater peripheral E-CR1 consumption in SLE patients presenting with renal flare [14]. Reduced levels of E-CR1 may reflect disease activity in lupus patients (associated with level of circulating ICs). However, in SLE patients also presenting with CRF, E-CR1 levels could not be used as a biomarker for the evaluation of disease activity or diagnosis because our data did not indicate any significant reduction in E-CR1 levels in these patients when compared with healthy controls $(3.69 \pm 0.31$ versus $3.82 \pm 0.32, P=0.5)$.

In patients with other autoimmune disorders such as MCTD, the use of sMFI values for E-CR1 as a diagnostic marker of SLE would not be viable because a reduction in E-CR1 levels was also found in this disorder (Table 2). MCTD is a systemic autoimmune disease with manifestations including polyarthritis, Raynaud's phenomenon, sclerodactyly, swollen hands and myopathy. Renal involvement with hypocomplementemia is one of major complication in MCTD [20]. Significant reduction of E-CR1 could be observed in our result and might be due to the involvement of complement systemic with formation of ICs in MCTD. A previous study revealed the mean number of E-CR1 is significantly lower in RA patients than in healthy controls [14]. Circulating CR1 can inhibit the differentiation of B cells and production of immunoglobulin [21]. In our study, a reduction in E-CR1 levels was also found in RA patients, but these were higher than in SLE patients. Except for poor differentiation with MCTD, our results indicated E-CR1 was still an effective diagnostic biomarker for SLE among autoimmune disorders, including RA, SS, APS, RD and AS. In patients with TB, consumption of E-CR1 antigens occurs. This prevents SLE from being differentiated from this infectious condition on the basis of E-CR1 levels. In the peripheral circulation, E-CR1 is an important mediator involved in the clearance of ICs; therefore, the degree of the reduction in levels of E-CR1 is likely to vary in disorders caused by abnormal deposition of circulating ICs [22-24].

In summary, our study supported the conclusion that reduction in E-CR1 levels was not absolutely specific for SLE diagnosis. Reduction of circulating E-CR1 was also observed in other autoimmune diseases (RA and MCTD) and non-autoimmune disease (TB). Reductions in E-CR1 represented a transient phenomenon associated with the levels of circulating ICs. However, it could be a useful parameter for evaluating disease activity as well as for assessment of renal involvement in SLE.

\section{Acknowledgements}

This study was supported by Taichung Armed-Forces General Hospital (grant no: $102-7$ and 103A07).

\section{References}

1. Klickstein LB, Bartow TJ, Miletic $V$, Rabson LD, Smith JA, et al. (1988) Identification of distinct $\mathrm{C} 3 \mathrm{~b}$ and $\mathrm{C} 4 \mathrm{~b}$ recognition sites in the human $\mathrm{C} 3 \mathrm{~b} / \mathrm{C} 4 \mathrm{~b}$ receptor (CR1, CD35) by deletion mutagenesis. J Exp Med 168: 1699-1717.

2. Klickstein LB, Barbashov SF, Liu T, Jack RM, Nicholson-Weller A (1997) Complement receptor type 1 (CR1, CD35) is a receptor for C1q. Immunity 7 : 345-355.

3. Ghiran I, Barbashov SF, Klickstein LB, Tas SW, Jensenius JC, et al. (2000) Complement receptor $1 / \mathrm{CD} 35$ is a receptor for mannan-binding lectin. $\mathrm{J}$ Exp Med 192: 1797-1808.

4. Rowe JA, Rogerson SJ, Raza A, Moulds JM, Kazatchkine MD, et al. (2000) 
Citation: Yang DH, Chen CH, Wei CC, Cheng YW (2014) Expression of Complement Receptor Type 1 on Erythrocytes in Autoimmune Diseases. J Mol Biomark Diagn 5: 163. doi:10.4172/2155-9929.1000163

Page 6 of 6

Mapping of the region of complement receptor $(\mathrm{CR}) 1$ required for Plasmodium falciparum rosetting and demonstration of the importance of CR1 in rosetting in field isolates. J Immunol 165: 6341-6346.

5. Erdei A, Prechl J, Isaák A, Molnár E (2003) Regulation of B-cell activation by complement receptors CD21 and CD35. Curr Pharm Des 9: 1849-1860.

6. Gergely P Jr, Isaák A, Szekeres Z, Prechl J, Erdei A, et al. (2007) Altered expression of Fcgamma and complement receptors on B cells in systemic lupus erythematosus. Ann N Y Acad Sci 1108: 183-192.

7. Chen $\mathrm{CH}$, Ghiran I, Beurskens FJ, Weaver G, Vincent JA, et al. (2007) Antibody CR1-2B11 recognizes a non-polymorphic epitope of human CR1 (CD35). Clin Exp Immunol 148: 546-554.

8. de Carvalho Lins CE, Pereira Crott LS, Teixeira JE, Barbosa JE (2004) Reduced erythrocyte complement receptor type 1 in systemic lupus erythematosus is related to a disease activity index and not to the presence or severity of renal disease. Lupus 13: 517-521.

9. lida K, Mornaghi R, Nussenzweig V (1982) Complement receptor (CR1) deficiency in erythrocytes from patients with systemic lupus erythematosus. J Exp Med 155: 1427-1438.

10. Singh V, Mahoney JA, Petri M (2008) Erythrocyte C4d and complement receptor 1 in systemic lupus erythematosus. J Rheumatol 35: 1989-1993.

11. Marzocchi-Machado CM, Alves CM, Azzolini AE, Polizello AC, Carvalho IF, et al. (2005) CR1 on erythrocytes of Brazilian systemic lupus erythematosus patients: the influence of disease activity on expression and ability of this receptor to bind immune complexes opsonized with complement from normal human serum. J Autoimmun 25: 289-297.

12. Ross GD, Yount WJ, Walport MJ, Winfield JB, Parker CJ, et al. (1985) Diseaseassociated loss of erythrocyte complement receptors (CR1, C3b receptors) in patients with systemic lupus erythematosus and other diseases involving autoantibodies and/or complement activation. J Immunol 135: 2005-2014.

13. Inada Y, Kamiyama M, Kanemitsu T, Clark WS, Asai Y (1986) Relationships between $\mathrm{C} 3 \mathrm{~b}$ receptor (CR1) activity of erythrocytes and positive Coombs' tests. Ann Rheum Dis 45: 367-372.
14. Kumar A, Malaviya AN, Srivastava LM (1994) Lowered expression of C3b receptor (CR1) on erythrocytes of rheumatoid arthritis patients. Immunobiology 191: 9-20.

15. Senbagavalli $P$, Geetha ST, Karunakaran K, Banu Rekha VV, Venkatesan $P$, et al. (2008) Reduced erythrocyte CR1 levels in patients with pulmonary tuberculosis is an acquired phenomenon. Clin Immunol 128: 109-115.

16. Manderson AP, Botto M, Walport MJ (2004) The role of complement in the development of systemic lupus erythematosus. Annu Rev Immunol 22: 431456.

17. Sturfelt G, Truedsson $L$ (2005) Complement and its breakdown products in SLE. Rheumatology (Oxford) 44: 1227-1232.

18. Arora V, Verma J, Dutta R, Marwah V, Kumar A, et al. (2004) Reduced complement receptor 1 (CR1, CD35) transcription in systemic lupus erythematosus. Mol Immunol 41: 449-456.

19. Manzi S, Navratil JS, Ruffing MJ, Liu CC, Danchenko N, et al. (2004) Measurement of erythrocyte $\mathrm{C} 4 \mathrm{~d}$ and complement receptor 1 in systemic lupus erythematosus. Arthritis Rheum 50: 3596-3604.

20. Ortega-Hernandez OD, Shoenfeld $Y$ (2012) Mixed connective tissue disease: an overview of clinical manifestations, diagnosis and treatment. Best Pract Res Clin Rheumatol 26: 61-72.

21. Kremlitzka M, Polgár A, Fülöp L, Kiss E, Poór G, et al. (2013) Complement receptor type 1 (CR1, CD35) is a potent inhibitor of B-cell functions in rheumatoid arthritis patients. Int Immunol 25: 25-33.

22. Khera R, Das N (2009) Complement Receptor 1: disease associations and therapeutic implications. Mol Immunol 46: 761-772.

23. Frieri M (2013) Mechanisms of disease for the clinician: systemic lupus erythematosus. Ann Allergy Asthma Immunol 110: 228-232.

24. Manzi S, Ahearn JM, Salmon J (2004) New insights into complement: a mediator of injury and marker of disease activity in systemic lupus erythematosus. Lupus 13: $298-303$ 